\title{
Hypsarrythmia - Do Age Have Any Relevance
}

\author{
Kamlesh Jha, Abhilasha Mishra*, Yogesh Kumar, Ramji Singh
}

\section{Kamlesh Jha, Abhilasha Mishra*, Yogesh Kumar, Ramji Singh}

Department of Physiology, All India Institute of Medical Sciences, Patna, Bihar, INDIA.

\section{Correspondence}

\section{Dr. Abhilasha Mishra}

Senior Resident, Department of Physiology, All India Institute of Medical Sciences, Patna, Bihar, INDIA.

Phone: +91-9140683829

Email: abhilashaabhi09@gmail.com

History

- Received: 18-08-2018

- Revised: 29-09-2018;

- Accepted: 05-10-2018.

DOI : 10.5530/ijcep.2018.5.3.11

\section{Copyright}

(c) 2018 Phcog.Net. This is an openaccess article distributed under the terms of the Creative Commons Attribution 4.0 International license.

\begin{abstract}
Hypsarrhythmia is a classical electroencephalographic finding in patients of infantile spasm and related syndromes like West syndrome and Otahara syndrome. It usually develops in early infancy and persists up to age of two years, after that it gets completely resolved or gets converted into other kinds of epilepsy. Persistence of hypsarrhythmia beyond this age group has rarely been reported in literature. In the present article we have noticed that hypsarrhythmia is present in older age group and it has also been noticed that these patients have first episode of seizure after the age of three years. Exact pathophysiology behind persistence of hypsarrhythmic pattern in patient more than 3 years and new onset of hypsarrhythmic pattern in older patients on electro encephalography is not known. Although it has been suggested that immaturity of thalamocortical synaptic pathway is responsible for hypsarrhythmia in infantile spasm.
\end{abstract}

Key words: Hypsarrhythmia, Electroencephalography, Infantile spasm, Older children, epilepsy,

\section{INTRODUCTION}

Seizure or fits and epilepsy of any kind is a unavoidable problem of the world. Around $5-10 \%$ of the total population of world had experienced these kind of episodes, at least once in their lifetime. Convulsion disorder affects $4-5 \%$ children around the world. Although incidence of convulsion disorder is comparatively higher in developing countries than in developed countries, but the exact reason behind this is unknown. Convulsion disorders in children affects the overall development of a child and thus affecting the wellbeing of society and most of the time it leaves a permanent neurological disability.

Infantile spasm is one of the highly morbid convulsive condition affecting children at very young age. Onset of this condition is usually occurs in early infancy. Typical EEG finding found in infantile spasm is high voltage chaotic background with multifocal spikes, commonly known as hypsarrhythmia. Usually hypsarrhythmia persists up to 2 year. After that it gets either completely resolved or gets converted into other EEG patterns. Persistence of hypsarrhythmia beyond three years or finding hypsarrhythmia in older age groups is a rare finding. If it is present in older children, it is usually associated with poor prognosis.

\section{What is Hypsarrythmia?}

Hypsarrythmia is derived from greek word, Hypsi, meaning high or mountainous and arrhythmia, meaning lack of rhythm. It is defines as chaotic high amplitude background with multifocal spikes in electroencephalography. ${ }^{[1]}$ Many clinicians specify a

minimum amplitude of $200 \mu \mathrm{V}$ to designate an activity as hypsarrythmic one. (Figure 1)

Hypsarrythmia is a classical EEG finding in infantile spasm. It is also an important component of West syndrome, consists of triad of infantile spasm, EEG finding of Hypsarrythmia and mental retardation. ${ }^{[2]}$ In general, Hypsarrythmia, an interictal finding in EEG is seen in patients of infantile spasm until the age of two tears, with onset usually in infancy (peak age of onset is 4-6 months). ${ }^{[3]}$ Finding Hypsarrythmia beyond the age of three years is a very rare finding. If it is present beyond this, it generally represents poor prognosis.

Objective of this review article is to find out various theories behind this rare finding.

\section{DYNAMICITY OF HYPSARRYTHMIA}

Nature of hypsarrythmia is very dynamic. It is an encephalographic pattern which can evolve from other patterns (e.g. from bursts suppression, like in otahara syndrome. ${ }^{[4]}$ Patients presenting with generalized spike wave abnormality in EEG, tends to develop hypsarrythmic pattern with progression of time.

On the other hand, the classical hypsarrythmic pattern can evolve into other epileptic patterns or can completely normalized.

\section{MODIFIED HYPSARRYTHMIA}

Typical hypsarrythmic pattern that is chaotic, high amplitude disorganized background of slow waves with multifocal spikes, is not commonly seen. 


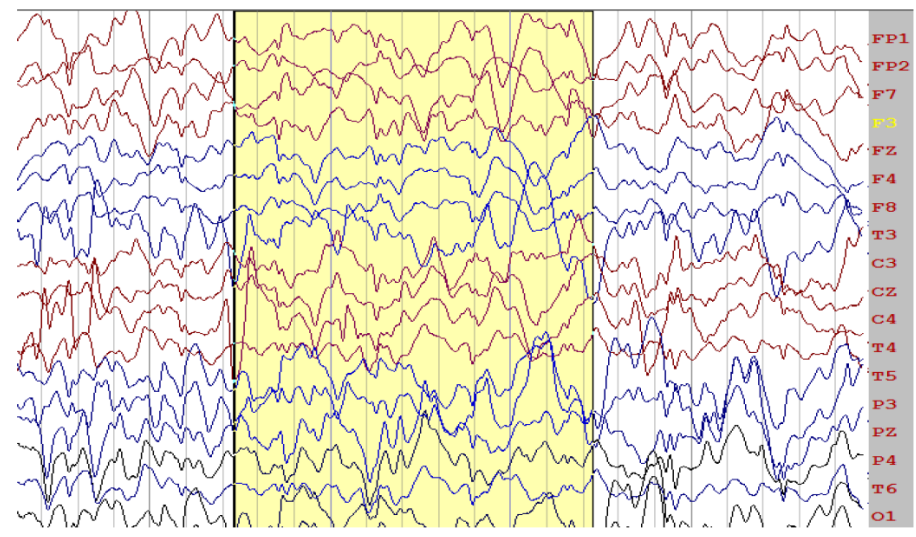

Figure 1: A pattern of hypsarrhythmia showing typical multifocal spikes with high voltage slow waves in a child of 6 years age.

Instead an atypical form of Hypsarrythmia is noticed in most of the neurophysiology lab. This is called as Modified Hypsarrythmia.

Modified Hypsarrythmia can be seen in these forms -

- 1-Hypsarrhythmia with increased interhemispheric synchronization. Characterized by symmetric and synchronized activity, seen in patients with longstanding evolution, especially in those with West syndrome that changes to Lennox-Gastaut syndrome.

- 2-Asymmetric hypsarrhythmia. Associated with a brain structural abnormality and does not necessarily predict the affected hemisphere.

- 3-Hypsarrhythmia with a consistent focus of abnormal discharge.

- 4-Hypsarrhythmia with episodes of voltage attenuation. Commonly seen during nonrapid eye movement (NREM) sleep. When the episodes of voltage attenuation appear at the same time as an epileptic spasm does, they are called electrodecrements.

- 5-Hypsarrhythmia with little spike or sharp activity. ${ }^{[5]}$

Various scoring system have been used to define and assess severity of hypsarrhythmia, in which Kramer scoring system is frequently used in various articles. In this scoring system items scored included disorganization, delta activity, amplitude and spike activity with scores from 0 to 3 each. In addition, a score of 1 each was given to any of the following additional patterns: electrodecremental response, burst suppression, absence of sleep pattern and relative normalization. Maximum score according to this reaches $16 .{ }^{[6]}$

One more scoring system to define and assess severity of hypsarrythmia, is BASED (Burden of Amplitudes and Epileptiform Discharges) scoring system. It is comparatively newer and objective type of scoring system. It includes number of multifocal spikes and amplitude of background slow waves in a one second bins. Based on this, scores 1 to 5 has been given. In this score 4 and 5 has been classified as hypsarrythmia. There is no any association found between higher scores and severity of disease in BASED scoring system. ${ }^{[7]}$

\section{Hypsarrythmia in older children}

Hypsarrythmia beyond the age of 3 years or finding hypsarrythmia as new in older age group is a rare finding. Presence of this finding in older age groups is an alarming sign. It might represent a poor prognosis due to some underlying neuronal developmental anomaly. A long term follow up study conducted by K. Kandan et al. (2011) described the finding of Hypsarrhythmia in children above 3 years.

This was a 5 years follow up from 2002 to 2007 they found that etiological factor behind persisted Hypsarrythmia is a severe maldevelopment of brain including leisencephaly, mitochondrial encephalopathy and hypoxic ischemic encephalopathy.

Another study conducted by Gibbs et al. in 1954 also concluded same finding in 3 year follow up of 237 patients of infantile spasm. They found Hypsarrythmia in 30 Patients, 21 of them between 3-4 years and 9 of them are above 5 years. ${ }^{[8]}$ Jevons et al. in 1970 also supported this finding. In their study, out of 68 children, 3 children were reported to have persistent hypsarrythmia. ${ }^{[9]}$ In yet another long term follow up study by Jeevon et al. (1973), done to evaluate the long term prognosis in West syndrome. Out of 106 patients, 3 patients had persistent hypsarrythmia in EEG, but they didn't reported the exact age of these patients. ${ }^{[10]}$

A retrospective study by Menezes and Rho concluded in their study, that 4 out of 26 children having persistent Hypsarrythmia. Etiologic factors reported in these studies included severe grade of migrational abnormalities, perinatal complications, genetic and mitochondrial abnormalities ${ }^{[11]}$ etc.

\section{Neuronal network involved}

It has been observed that development of seizure disorder in pediatric age group is more likely due to loss of inhibition over excitatory fibers.

Usually inhibitory fibers are enough to counterbalance the excitatory activity of neurons.

Epileptiform discharge within Hypsarrythmia are associated with hemodynamic and metabolic change in cortex while high voltage slow waves are produced from cortex and subcortical structure. ${ }^{[12]}$ Additionally slow waves also accompanies a activation in putamen brain stem and thalamus. Subcortical structure appear to play more important role in generating slow waves. (Figure 2).This study hypothesized that clinical seizures might result from intermittent interference of descending brainstem pathways controlling spinal reflex activities.

Hypsarrythmia pattern in EEG are produced probably due to the ascending pathway from the same brainstem areas that projected widely to cerebral cortex. ${ }^{[13]}$

Raphe striatal and reticulostriatal pathways of basal ganglia are also apparently involved in the pathogenesis of this EEG pattern. ${ }^{[14]}$

\section{Prognostication of hypsarrythmia in older children}

Most of the older children with electroencephalographic diagnosis of hypsarrythmia usually have complaint of persistent seizure, mostly refractory to antiepileptic regimens. Hypsarrythmic children with epileptic encephalopathies may show some response to certain class of antiepileptic medications but compared to younger children, they are distinctly less responsive.

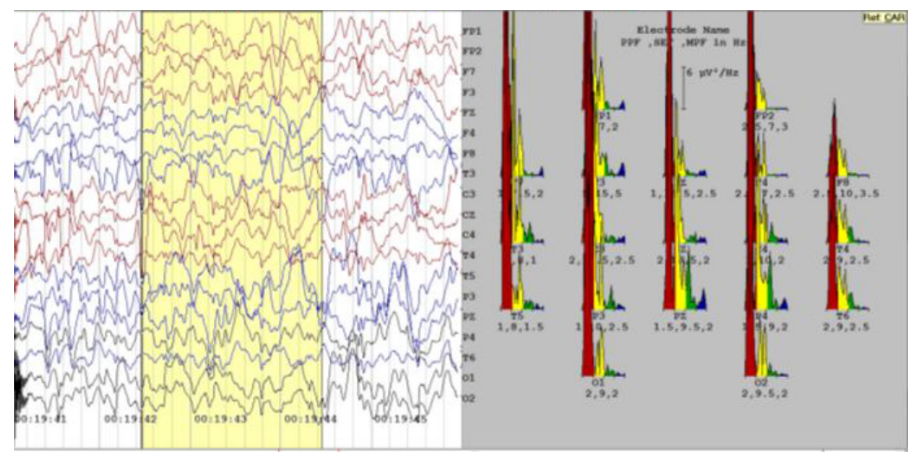

Figure 2: Hypsarrythmic pattern showing predominance of delta waves in a segregated image, in a 6 year old boy. Electroencephalographic recording of the was done in neurophysiology lab of Physiology department, All India Institute of medical sciences, patna. 
Fewer if any study has been done to compare the prognostic factors and outcome of hypsarrythmia in younger age group children to that of children with age more than 3 years. Though pieces of evidences suggest that most of the therapeutic modalities suggested for infantile spasm (hysparrythmia) including hormone therapy are more effective in the younger age children. ${ }^{[15]}$

Here in these scenario in which hypsarrythmia has been observed in older age groups, indicates immature neural developments of subcortical areas and corticothalamic pathways. We need to focus on the etiological factors involved in these cases as there are varied etiological factors and treatment differs with each factor involved.

\section{CONCLUSION}

- Persistence of Hypsarrythmia in EEG is a rare finding.

- Mean age of disappearance of Hypsarrythmia pattern is 2 years and it developed in early infancy.

- Many studies conducted in previous years has supported this elctroencephalographic finding beyond the age of 3 years.

- It has also been suggested that the etiology behind persistence of this pattern is severe brain malformation and developmental anomalies.

- Children having persisted Hypsarrythmia pattern in EEG are more likely to develop developmental delay and delayed psychomotor responses.

- So, the patient having persistent Hypsarrythmia should be assessed thoroughly for vast categories of etiologies like development disorder mitochondrial encephalopathy's perinatal complication hypoxic ischemic encephalopathy and treatment based on etiology and clinical presentation.

Finding hypsarrythmia in older age groups suggest that the neuronal immaturity is still persisting beyond a definite age. There is immature connections between subcortical structures and corticothalamic pathways, which should get matured at the age of three years maximum.

Area of focus in these cases are basically etiology as the treatment differs. Pharmacological treatment available are no much promising till date.

So we should also think of some non pharmacological modality for these children to attain socially compatible life.

\section{ACKNOWLEDGEMENT}

I acknowledge all the staff workers of neurophysiology lab AIIMS Patna, for their hard work and kind support.

\section{CONFLICT OF INTEREST}

The authors declare no conflict of interest.

\section{ABBREVIATIONS}

EEG: Electroencephalography; NREM: Non Rapid Eye Movement Sleep.

\section{REFERENCES}

1. Gibbs EL, Fleming MM, Gibbs FA. Diagnosis and prognosis of hypsarhythmia and infantile spasms. Pediatrics. 1954;13(1):66-73.

2. Wong M, Trevathan E. Infantile spasms. Pediatr Neurol. 2001;24:89-98.

3. Ohtahara S, Ohtsuka Y, Yamatogi Y. The West syndrome: developmental aspects. Acta Paediatr Jpn. 1987;29(1):61-9.

4. Ohtahara S, Ohtsuka Y, Yamatogi Y. The West syndrome: developmental aspects. Acta Paediatr Jpn. 1987;29(1):61-9.

5. Hrachovy RA, Frost JD, Kellaway P. Hypsarrhythmia: variations on the theme. Epilepsia. 1984;25(3):317-25.

6. Kramer U, Sue WC, Mikati MA. Hypsarrhythmia: frequency of variant patterns and correlation with etiology and outcome. Neurology. 1997;48(1):197-203.

7. Mytinger JR, Hussain SA, Islam MP, Millichap JJ, Patel AD, Ryan NR, et al. Article title? Epilepsy Res. 2015;116:93-8. doi: 10.1016/j.eplepsyres.2015.07.008.

8. Gibbs EL, Fleming MM, Gibbs FA. Diagnosis and prognosis of hypsarhythmia and infantile spasms. Pediatrics. 1954;13(1):66-73.

9. Jeavons PM, Bower BD, Dimitrakoudi M. Long-term prognosis of 150 cases of West syndrome. Epilepsia. 1973;14(2):153-64.

10. Jeavons PM, Harper JR, Bower BD. Long-term prognosis in infantile spasms: a follow-up report on 112 cases. Dev Med Child Neurol. 1970;12(4):413-21.

11. de Menezes MA, Rho JM. Clinical and electrographic features of epileptic spasms persisting beyond the second year of life. Epilepsia. 2002;43(6):623-30.

12. Michael S, Giuseppe C. Functional neuroimaging in epileptic encephalopathies. Epilepsia. 2013;54(s8):27-33.

13. Juhasz C, Chugani HT, Muzik O, Chugani DC. Neuroradiological assessment of brain structure and function and its implication in the pathogenesis of West syndrome. Brain Dev. 2001;23(7):488-95.

14. Hayashi M. Neuropathology of the limbic system and brainstem in West syndrome. Brain Dev. 2001;23(7):516-22.

15. Sharma S, Sankhyan N, Gulati S, Agrawala A. Use of the modified Atkins diet in infantile spasm refractory to first line treatment. Seizure. 2012;21(1):45-8.

Cite this article: Jha K, Mishra A, Kumar Y, Singh R. Hypsarrythmia - Do Age Have Any Relevance. Int J Clin Exp Physiol.

2018;5(3):156-8. 